\title{
A Lagrangian Model for Hardening Behaviour of Materials at Finite Deformation based on the Right Plastic Stretch Tensor
}

\author{
Amin Eshraghi ${ }^{\dagger}$ \\ ${ }^{\dagger}$ Corresponding Author, Department of Mechanical and Mechatronics Engineering, University of Waterloo, \\ Waterloo, Ontario, N2L 3G1, Canada \\ Email: maeshrag@uwaterloo.ca
}

Tel. +1 $519888-4567$ x 37493

Fax. +1 $519885-58623$

Hamid Jahed

Professor, Department of Mechanical and Mechatronics Engineering, University of Waterloo, Waterloo, Ontario, N2L 3G1, Canada

Email: hjahedmo@uwaterloo.ca

\section{Steve Lambert}

Professor, Department of Mechanical and Mechatronics Engineering, University of Waterloo, Waterloo, Ontario, N2L 3G1, Canada

Email: steve@uwaterloo.ca 


\begin{abstract}
In this paper a modified multiplicative decomposition of the right stretch tensor is proposed and used for finite deformation elastoplastic analysis of hardening materials. The total symmetric right stretch tensor is decomposed into a symmetric elastic stretch tensor and a non-symmetric plastic deformation tensor. The plastic deformation tensor is further decomposed into an orthogonal transformation and a symmetric plastic stretch tensor. This plastic stretch tensor and its corresponding Hencky's plastic strain measure are then used for the evolution of the plastic internal variables. Furthermore, a new evolution equation for the back stress tensor is introduced based on the Hencky plastic strain. The proposed constitutive model is integrated on the Lagrangian axis of the plastic stretch tensor and does not make reference to any objective rate of stress. The classic problem of the simple shear is solved using the proposed model. Results obtained for the problem of simple shear are identical to those of the selfconsistent Eulerian rate model based on the logarithmic rate. Furthermore, extension of the proposed model to the mixed nonlinear isotropic/kinematic hardening behaviour is presented. The model is used to predict the nonlinear hardening behaviour of SUS 304 stainless steel under fixed-end finite torsional loading. Results obtained are in good agreement with the available experimental results reported for this material under fixed-end finite torsional loading.
\end{abstract}

Keywords: Finite deformation; Multiplicative decomposition; Mixed hardening 


\section{1- Introduction}

Small strain elastoplastic formulation of metals and polycrystalline solids based on the phenomenological plasticity models uses an additive decomposition of the total strain into its elastic and plastic parts [1,2]. However, such decomposition is no longer valid if material is subjected to finite deformation loading path [1]. The so-called swift effect [3], which happens in finite torsional loading of fixed cylindrical bars, is one of the cases where infinitesimal plasticity models fail to give proper predictions. An extension of the classical infinitesimal plasticity to finite deformation analysis requires proper decomposition of the deformation into its elastic and inelastic parts. Two classes of decomposition have been widely used in the literature of finite deformation plasticity $[1,4]$. The first one is mainly based on the additive decomposition of the strain rate tensor while the second one uses a multiplicative decomposition of the deformation gradient.

Green and Naghdi [5] introduced a general framework of finite elastoplasticity using the thermodynamics of the continua. An additive decomposition of the Green-Lagrange strain tensor into elastic and plastic parts was used in their formulation. However, the decomposition used did not necessarily represent the plastic part of the deformation if the first part was assumed to be the exact elastic part of deformation. Recently, Sansour and Wagner [6] used an additive decomposition of the Hencky's strain tensor. The plastic flow rule and the related internal plastic variables and their corresponding thermodynamics driving forces were based on the conjugate measure of the stress to the Hencky (logarithmic) strain.

Constitutive models based on the additive decomposition of the strain rate tensor into elastic and inelastic parts have been mostly employed for setting up an Eulerian rate formulation of elastoplasticity at finite deformation [7-10]. A hypoelastic constitutive model relates the elastic part of the strain rate tensor to an objective rate of the Kirchhoff stress tensor while the plastic part is updated based on a specified flow rule similar to that of the infinitesimal plasticity. This class of Eulerian rate formulation entails use of objective quantities and their corresponding objective rates [11,12]. Certain issues such as shear oscillation in the problem of simple shear $[11,13]$ and dissipation in closed path elastic loading [14] were observed when different objective rates of stress were used in the hypoelastic model. Sources of such issues have been associated with hypoelastic model non-integrability as a Cauchy and Green elastic material (see for example references $[11,15,16])$. To resolve such issues, following the work of Lehmann et al. [17], 
Reinhardt and Dubey [18], and Xiao et al. [19], a new objective rate of stress, called the D or logarithmic rate, was introduced. Furthermore, Bruhns et al. [10] investigated the integrability conditions of the classical hypoelastic model and showed that the grade zero hypoelastic model is unconditionally integrable as a Cauchy and Green elastic material when the logarithmic (D) rate is used in the model. Based on this, Bruhns et al. [10] developed a self-consistent Eulerian rate form of elastoplasticity for the finite deformation analysis of hardening materials using the logarithmic (D) rate.

The class of constitutive models using the multiplicative (Lee) decomposition [20] of the deformation gradient into its elastic and inelastic parts has also been physically well grounded based on crystal plasticity observations [4,21,22]. Simo [23] and Simo and Ortiz [24] used a multiplicative decomposition of the deformation gradient and derived equivalent representations for finite elastoplastic formulations in material (convected) and spatial frameworks. A hyperelastic function was used to relate the Lagrangian measure of the strain to the symmetric Piola-Kirchhoff stress tensor in the material representation of the formulation. Similarly, a hyperelastic function was used to relate the spatial metric tensor to the Kirchhoff stress tensor. The requirement of spatial covariance entailed use of the Lie derivative of the Kirchhoff stress in the spatial formulation. The plastic flow was derived based on the assumption of maximum plastic dissipation similar to the case of infinitesimal plasticity. Furthermore, following the volumetric/deviatoric decoupling of the deformation gradient used in [25,26], Simo [23] and Simo and Ortiz [24] derived a decoupled volumetric/deviatoric response of their hyper-based constitutive model and showed that the general return mapping algorithm widely used in infinitesimal plasticity [4] was valid for their model integration [27]. Recent formulations based on the class of multiplicative decomposition use a hyperelastic strain energy function and relate the rotated Kirchhoff stress to the Hencky strain for the case of isotropic plasticity [28-30]. Such hyperelastic function has been shown to provide good prediction for moderately large elastic strains in metal plasticity [31,32]. Simple algorithmic implementation of this class of constitutive models for the case of isotropic plasticity has made this class of models more popular in recent numerical implementations $[29,30]$. Furthermore, the general return mapping integration method used in algorithmic implementation of the infinitesimal plasticity models can still be used for the numerical integration of this class of constitutive models [4]. Use of a "total hyperelastic" relation bypasses the need for objective rates of stress and as a result the need for a neutrally 
objective integration algorithm is eliminated [4]. Furthermore, since the model is based on the existence of a hyperelastic function and is consistent with the notion of Cauchy and Green elasticity, issues regarding the model non-integrability as found in "hypo-based" Eulerian rate formulation do not appear in "hyper-based" formulation of elastoplasticity.

The multiplicative decomposition has also been used in Eulerian rate formulation of elastoplasticity. Metzger and Dubey [33] decomposed the left stretch tensor into a symmetric elastic and non-symmetric plastic parts. Based on this decomposition, a modified additive decomposition of the stretching tensor into elastic and plastic parts was derived. The hypoelastic model based on the modified elastic strain rate was integrated for different objective rates of stress on the principal axis of the elastic left stretch tensor for the case of isotropic $J_{2}$ plasticity. Results obtained were identical for all of the objective rates used in their modified model. More recently, Ghavam and Naghdabadi [34] used a modified decomposition of the Metzger and Dubey decomposition [33] for linear kinematic hardening and mixed hardening behaviours under simple shear. They showed that results were slightly affected by the type of decomposition used in their integration process for different objective rates of stress.

In this paper a new constitutive model based on the multiplicative decomposition of the right stretch tensor is introduced. The symmetric right stretch tensor is successfully decomposed into a symmetric elastic and a non-symmetric inelastic part. The rotated Kirchhoff stress is related to the Lagrangian elastic Hencky strain. The non-symmetric plastic tensor is further decomposed into an orthogonal transformation and a pure plastic stretch. Using the method of principal axes and transferring all the tensor variables onto the Lagrangian axis of the symmetric plastic stretch tensor, the model is successfully integrated with a new evolution equation for the back stress tensor. Results obtained for the problem of simple shear are identical to those of the selfconsistent Eulerian rate model introduced by Bruhns et al. [10] in which an additive decomposition of the strain rate tensor and the logarithmic rate of the Kirchhoff stress were used. Furthermore, the model is extended to predict the mixed nonlinear hardening behaviour of SUS 304 stainless steel under fixed-end finite torsional loading. Predicted results are in good agreement with those of the experimental results reported by Ishikawa [35]. 


\section{2- Continuum formulation of elastoplasticity}

Assuming a particle of a deforming continuum with the initial position vector $X=\varphi(X, 0)$ at $t=0$ and current position vector $x=\varphi(X, t)$, the deformation gradient $F$ can be defined as follows:

$F=\left[\frac{\partial x_{i}}{\partial X_{j}}\right]=\left[\frac{\partial \varphi_{i}(X, t)}{\partial X_{j}}\right]$

A polar decomposition of the deformation gradient results into a pure stretch and a rigid rotation of the deforming body:

$F=R U=V R$

in which $R$ is the orthogonal rigid rotation of the body and $U$ and $V$ are the right and left stretch tensors, respectively. The velocity gradient $l$ can be decomposed into its symmetric and skewsymmetric parts as follows:

$l=\left[\frac{\partial v_{i}}{\partial x_{j}}\right]=d+w$

in which $v$ is the velocity of the particle, $d$ and $w$ are the strain rate (rate of deformation) and the material spin tensors, respectively.

For an Eulerian rate formulation of elastoplasticity, the strain rate tensor $d$ can be additively decomposed into its elastic and inelastic parts $[4,7,8,10,18]$ :

$d=d^{e}+d^{p}$

Superscripts ${ }^{e}$ and ${ }^{p}$ refer to the elastic and plastic parts of a tensor, respectively. The elastic part of the strain rate tensor is related to an objective rate of the Kirchhoff stress through a hypoelastic constitutive model [11,36]:

$\stackrel{*}{\dot{\tau}}=M(\tau): d^{e}$

where $\tau$ represents the Kirchhoff stress, a superposed dot with a superscript ${ }^{*}$ indicates objective rate of the corresponding tensor in an $\Omega^{*}$ spinning frame, $M$ is the fourth order stress-dependent hypoelasticity tensor, and the operator “:” represents the dot product of tensor variables. Examples of objective rates are the well-known Jaumann and Green-McInnis-Naghdi rates measured in the $\Omega^{*}=\Omega^{J}=w$ and $\Omega^{*}=\Omega^{G M N}=\dot{R} R^{T}$ spinning frames [4,37], respectively.

The plastic part of the strain rate tensor can be related to the shift stress tensor by specifying a proper flow rule. Use of the additive decomposition of the strain rate tensor in the constitutive model of elastoplasticity requires that the constitutive model given by (5) be integrable in the 
sense of Cauchy and Green elasticity [11,15,26,38]. Elastic integrability conditions of rate model in equation (5) enforce the existence of a hyperelastic strain energy function for which equation (5) is derivable. In other words, for a physically acceptable additive decomposition of strain rate tensor given by equation (4), equation (5) should result into an additive decomposition of the total stress power into its elastic and plastic parts [38]. It has been shown that the logarithmic (D) rate of stress along with a grade zero hypoelastic model of equation (5), i.e. the hypoelastic model with a constant hypoelasticity tensor, yield an unconditionally integrable model which can be used with the additive decomposition given by (4) for setting up a self-consistent Eulerian rate form of elastoplasticity [10].

On the other hand, a multiplicative decomposition of the deformation gradient into elastic and plastic parts can also be used as follows [20]:

$F=F^{e} F^{p}$

This decomposition is based on the assumption of an intermediate stress free configuration as shown in figure (1). In this figure " $n$ " and " $n+1$ " refer to any two consecutive configurations at time $\mathrm{t}$ and $\mathrm{t}+\Delta \mathrm{t}$, respectively.

\section{Figure 1- Schematic representation of the multiplicative decomposition}

Using the relation $l=\dot{F} F^{-1}$ and taking the time derivative of equation (6), a modified additive decomposition can be found as follows:

$l=\dot{F} F^{-1}=l^{e}+F^{e} \bar{L}^{p} F^{e^{-1}}=l^{e}+l^{p}$

in which $l^{e}=\dot{F}^{e} F^{e-1}$ is the spatial elastic part of the velocity gradient, $\bar{L}^{p}=\dot{F}^{p} F^{p^{-1}}$ is the plastic part of the velocity gradient on the intermediate plastic configuration, the term $l^{p}$ is the push-forward of the plastic part of the velocity gradient on the current configuration, and a superposed bar indicates variables on the intermediate configuration. Equation (7) defines a new type of additive decomposition for the velocity gradient as compared to equation (4). It is clear that unlike decomposition given by (4) which is on the current Eulerian configuration, the decomposition given by (7) associates with two different configurations for the elastic and plastic part of the strain rate tensor. Pulling back equation (7) on the intermediate configuration gives:

$\bar{L}=F^{e^{T}} l F^{e}=F^{e^{T}} l^{e} F^{e}+F^{e^{T}} F^{e} \bar{L}^{p} F^{e^{-1}} F^{e}=\bar{L}^{e}+\bar{C}^{e} \bar{L}^{p}$ 
in which $\bar{L}$ is the modified velocity gradient on the intermediate configuration and $\bar{C}^{e}$ is the right elastic Cauchy-Green tensor on the intermediate configuration. Pull-back and push-forward of kinematics and kinetics tensor quantities are discussed in details in $[4,39]$ and references therein.

Defining $\bar{S}=F^{e^{-1}} \tau F^{e^{-T}}$ as the pull-back of the Kirchhoff stress tensor on the intermediate configuration, the stress power can be written as follows:

$\dot{W}=\tau: l=\bar{S}: \bar{L}=\bar{S}:\left(\bar{L}^{e}+\bar{C}^{e} \bar{L}^{p}\right)=\bar{S}:\left(\bar{d}^{e}+\bar{w}^{e}\right)+\bar{S}: \bar{C}^{e}\left(\bar{d}^{p}+\bar{w}^{p}\right)$

The modified elastic spin $\bar{w}^{e}$ produces no work on the intermediate configuration due to the symmetry of $\bar{S}$. The non-symmetric Mandel stress tensor [40] is then defined by $\bar{\Gamma}=\bar{C}^{e} \bar{S}$ and is decomposed into symmetric and skew-symmetric parts. The modified stress power will therefore be:

$\dot{W}=\bar{S}: \bar{d}^{e}+\bar{\Gamma}_{\text {sym }}: \bar{d}^{p}+\bar{\Gamma}_{\text {skew }}: \bar{w}^{p}$

which shows that the symmetric part of the Mandel stress tensor generates power on the modified plastic strain rate while the skew-symmetric part of it generates power on the modified plastic $\operatorname{spin}[30]$.

Assuming isotropic elasticity for which the principal axes of the stress and elastic stretch coincide, the eigenvectors of $\bar{S}$ and $\bar{C}^{e}$ coincide and as a result the skew-symmetric part of the Mandel stress tensor vanishes, i.e. $\bar{\Gamma}_{\text {skew }}=0$. This means that the plastic spin does not dissipate energy during deformation. Furthermore, as a result of the commutative property of stress and elastic stretch and using the right polar decomposition of the elastic deformation gradient, i.e. $F^{e}=R^{e} U^{e}$, the symmetric Mandel stress tensor will be modified as follows:

$\bar{\Gamma}_{\mathrm{sym}}=\frac{1}{2}\left(\bar{C}^{e} \bar{S}+\bar{S} \bar{C}^{e}\right)=U^{e} \bar{S} U^{e}=R^{e T} \tau R^{e}=\bar{\tau}$

which shows that in isotropic plasticity the Mandel stress tensor is the same as the rotated Kirchhoff stress tensor [30].

Setting up the dissipation inequality and assuming a hyperelastic strain energy function which defines a linear relationship between the rotated Kirchhoff stress and the Hencky elastic strain on the intermediate configuration, Weber and Anand [28] and Gabriel and Bathe [29] defined the set of constitutive equations for the $J_{2}$ flow theory as follows:

$\bar{\Gamma}=\bar{\Gamma}_{\mathrm{sym}}=\bar{\tau}=\frac{\partial W}{\partial E^{e}}=M: E^{e}$ 
in which $E^{e}=\ln U^{e}$ is the Lagrangian Hencky (logarithmic) elastic strain tensor and $M$ is the isotropic fourth-order elasticity tensor. The following evolution equations for the plastic internal variables were also used for the plastic intermediate configuration update [29]:

$\dot{F}^{p}=\bar{L}^{p} F^{p} ; \bar{d}^{p}=\dot{\alpha} \frac{\partial \phi}{\partial \bar{\eta}} ; \phi=\sqrt{\frac{3}{2} \bar{\eta}: \bar{\eta}}-\kappa$

in which $\phi$ is the Mises plastic potential, $\dot{\alpha}$ is the consistency plastic multiplier, $\kappa$ is the current size of the yield surface, and $\bar{\eta}$ is the shift stress tensor. Details of numerical integration with the use of the exponential mapping algorithm for the stress and plastic intermediate configuration updates can be found in $[29,30]$.

\section{3- Proposed constitutive model of elastoplasticity}

In the Lagrangian formulation of elastoplasticity based on the multiplicative decomposition a right stretch decomposition is often used. However, polar decompositions based on the left stretch tensor may also be used in constitutive models which would result into an Eulerian rate formulation (see for example Metzger and Dubey [33], Ghavam and Naghdabadi [34], and Reinhardt and Dubey [41]). While the left stretch decomposition entails use of an objective rate of the Kirchhoff stress and requires a neutrally objective integration scheme, the right stretch decomposition uses a total relation between the rotated Kirchhoff stress and Hencky strain through a hyperelastic strain energy function and bypasses the need for objective rate quantities as discussed in the previous section. Multiplicative decomposition based on the right stretch tensor is employed here.

Assuming that the total symmetric right stretch tensor can be decomposed into a symmetric elastic part and a non-symmetric plastic part, one can write:

$F=R U=F^{e} F^{p}=R U^{e} \chi^{p}$

$U=U^{e} \chi^{p}$

The non-symmetric plastic tensor $\chi^{p}$ can further be decomposed into a symmetric plastic stretch tensor $U^{p}$ and an orthogonal transformation tensor $Q^{p}$ :

$\chi^{p}=Q^{p} U^{p}$

As a result, the modified plastic velocity gradient $\bar{L}^{p}$ can be obtained as follows:

$\bar{L}^{p}=\dot{\chi}^{p} \chi^{p^{-1}}=\dot{Q}^{p} Q^{p^{T}}+Q^{p}\left(\dot{U}^{p} U^{p^{-1}}\right) Q^{p^{T}}=\Omega^{\mathrm{Q}}+Q^{p}\left(\dot{U}^{p} U^{p^{-1}}\right) Q^{p^{T}}$

Figure (2) shows a schematic representation of the proposed decomposition: 
Figure 2- Schematic representation of the proposed multiplicative decomposition

In figure (2), the non-symmetric plastic deformation $\chi^{p}$ maps the old configuration " $n$ " onto the stress-free intermediate plastic configuration. This mapping induces no stress in the body and is assumed to be an isochoric mapping. The symmetric elastic stretch tensor then deforms the midconfiguration into a stressed body. Finally, the rigid rotation $R$ maps the stretched body onto the current configuration " $n+1$ ".

Since $\bar{L}^{p}=\bar{d}^{p}+\bar{w}^{p}$, equation (16) can be re-written as follows:

$\bar{d}^{p}=\frac{1}{2} Q^{p}\left(\dot{U}^{p} U^{p^{-1}}+U^{p^{-1}} \dot{U}^{p}\right) Q^{p^{T}}$

$\bar{w}^{p}=\Omega^{\mathrm{Q}}+\frac{1}{2} Q^{p}\left(\dot{U}^{p} U^{p^{-1}}-U^{p^{-1}} \dot{U}^{p}\right) Q^{p^{T}}$

With the rotation of the Lagrangian axis of the right plastic stretch tensor, $R_{L}^{p}$, the diagonalized form of the plastic stretch tensor can be obtained using the following transformation:

$U^{p}=\left(R_{L}^{p}\right)\left(\Lambda_{d}^{p}\right)\left(R_{L}^{p}\right)^{T}$

Similarly, the diagonalized plastic stretch tensor, $\Lambda_{d}^{p}$, can be rotated back to the left plastic stretch tensor using the rotation of the Eulerian axis as follows:

$V^{p}=\left(R_{E}^{p}\right)\left(\Lambda_{d}^{p}\right)\left(R_{E}^{p}\right)^{T}$

in which $R_{E}^{p}$ is the rotation of the Eulerian axis of the plastic stretch tensor and $V^{p}$ is the symmetric left plastic stretch tensor satisfying: $\chi^{p}=Q^{p} U^{p}=V^{p} Q^{p}$.

The relation between the Lagrangian and Eulerian axes is given as follows:

$R_{E}^{p}=Q^{p} R_{L}^{p}$

and the evolution equations for the orthogonal plastic rotation and its corresponding Lagrangian and Eulerian spins are given as follows:

$\dot{Q}^{p}=\Omega^{Q} Q^{p}$

$\dot{R}_{E}^{p}=R_{E}^{p} \Omega_{E}^{p}$

$\dot{R}_{L}^{p}=R_{L}^{p} \Omega_{L}^{p}$

in which $\Omega_{E}^{p}$ and $\Omega_{L}^{p}$ are the spins of the Eulerian and Lagrangian triads satisfying the equality $\Omega^{Q}=\left(R_{E}^{p}\right)\left(\Omega_{E}^{p}-\Omega_{L}^{p}\right)\left(R_{E}^{p}\right)^{T}$, respectively.

Transferring equation (17) to the Lagrangian axis of the plastic stretch tensor yields: 
$\bar{d}_{E}^{p}=\dot{\Lambda}_{d}^{p} \Lambda_{d}^{p-1}+\frac{1}{2}\left(\Lambda_{d}^{p-1} \Omega_{L}^{p} \Lambda_{d}^{p}-\Lambda_{d}^{p} \Omega_{L}^{p} \Lambda_{d}^{p-1}\right)$

$\bar{w}_{E}^{p}=\Omega_{E}^{p}-\frac{1}{2}\left(\Lambda_{d}^{p-1} \Omega_{L}^{p} \Lambda_{d}^{p}+\Lambda_{d}^{p} \Omega_{L}^{p} \Lambda_{d}^{p-1}\right)$

in which $\bar{d}_{E}^{p}=R_{E}^{p^{T}} \bar{d}^{p} R_{E}^{p}$ and $\bar{w}_{E}^{p}=R_{E}^{p^{T}} \bar{w}^{p} R_{E}^{p}$ are the Eulerian representations of the plastic strain rate and plastic spin, respectively. Following the method of the principal axes (see for example Reinhardt and Dubey [41], Hill [42], and Eterovic and Bathe [43]), the symmetric and skew-symmetric parts of the equation (22-1) give the following relations for the diagonalized plastic stretch tensor and its corresponding Lagrangian spin:

$\frac{\left(\dot{\Lambda}_{d}^{p}\right)_{i \mathrm{i}}}{\lambda_{i}^{p}}=\left(\bar{d}_{E}^{p}\right)_{i i} \quad ; \quad$ (no sum)
$\left(\Omega_{L}^{p}\right)_{i j}=\frac{2 \lambda_{j}^{p} \lambda_{i}^{p}}{\lambda_{j}^{p^{2}}-\lambda_{i}^{p^{2}}}\left(\bar{d}_{E}^{p}\right)_{i j} \quad ;(i \neq j)$

Similarly, use of equations (22-2) and (23-2) gives the following relation for the evolution of the Eulerian spin of the plastic stretch:

$\left(\Omega_{E}^{p}\right)_{i j}=\left(\bar{w}_{E}^{p}\right)_{i j}+\frac{\lambda_{j}^{p^{2}}+\lambda_{i}^{p^{2}}}{\lambda_{j}^{p^{2}}-\lambda_{i}^{p^{2}}}\left(\bar{d}_{E}^{p}\right)_{i j} ;(i \neq j)$

in which $\lambda^{p}$ 's are the principal plastic stretches.

The rotated Kirchhoff stress, $\bar{\tau}$, is work conjugate to the Lagrangian Hencky strain (see for example Gabriel and Bathe [29] and Hoger [44]) for the case of isotropic plasticity. Defining $\overline{\bar{\tau}}_{L}$ and $\overline{\overline{U^{e}}}{ }_{L}$ as the Lagrangian representation of the rotated Kirchhoff stress and elastic right stretch tensors on the Lagrangian triad, we have:

$$
\begin{aligned}
& \overline{\bar{\tau}}_{L}=\left(Q^{p} R_{L}^{p}\right)^{T} \bar{\tau}\left(Q^{p} R_{L}^{p}\right)=R_{E}^{p T} \bar{\tau} R_{E}^{p} \\
& \overline{\overline{U^{e}}}{ }_{L}=\left(Q^{p} R_{L}^{p}\right)^{T} \overline{U^{e}}\left(Q^{p} R_{L}^{p}\right)=R_{E}^{p T} \overline{U^{e}} R_{E}^{p}
\end{aligned}
$$

in which a superposed double bar along with a subscript ${ }_{L}$ indicate the components of a tensor on the Lagrangian axis of the plastic stretch tensor.

The Lagrangian rotated Kirchhoff stress, $\overline{\bar{\tau}}_{L}$, can be related to the Lagrangian rotated elastic Hencky strain through a hyperelastic function as follows:

$$
\overline{\bar{\tau}}_{L}=M:\left(R_{E}^{p^{T}} \ln \overline{U^{e}} R_{E}^{p}\right)=M: \ln \left(R_{E}^{p^{T}} \overline{U^{e}} R_{E}^{p}\right)=M: \ln {\overline{\overline{U^{e}}}}_{L}
$$


In equation (26) the fourth order elasticity tensor $M$ is assumed to be isotropic. Equation (26) defines the elastic part of the proposed constitutive model on the Lagrangian axis of the plastic stretch.

The shift stress tensor on the intermediate configuration $\bar{\eta}=\bar{\tau}^{\operatorname{dev}}-\bar{\beta}$, where $\bar{\beta}$ is the deviatoric back stress tensor and superscript ${ }^{\text {dev }}$ represents the deviatoric part of a symmetric tensor, can be rotated to the Lagrangian axis of plastic stretch as follows:

$\overline{\bar{\eta}}_{L}=\left(Q^{p} R_{L}^{p}\right)^{T} \bar{\eta}\left(Q^{p} R_{L}^{p}\right)=R_{E}^{p T} \bar{\eta} R_{E}^{p}=\overline{\bar{\tau}}_{L}^{\mathrm{dev}}-\overline{\bar{\beta}}_{L}$

The following evolution equation for the back stress tensor on the Lagrangian axis is proposed:

$\dot{\overline{\bar{\beta}}}_{L}=H{\dot{\overline{\bar{E}^{p}}}}_{L}$

Similar expressions can be proposed for a nonlinear back stress evolution equation which will be discussed in the next section. In equation (28), $H$ is the hardening modulus and $\overline{\overline{\bar{E}^{p}}}$ is the material time rate of the Lagrangian plastic Hencky strain and is related to the plastic strain rate tensor as follows:

$\left[\dot{\bar{E}}_{L}\right]_{i j}=h_{i j}^{\log }\left[\bar{d}_{E}^{p}\right]_{i j} ;($ no sum on $i$ and $j$ )

in which $h_{i j}^{\log }$ is the scaling function defined as:

$h_{i j}^{\log }= \begin{cases}1 & ; \text { if } \lambda_{j}^{p}=\lambda_{i}^{p} \\ \frac{2 \ln \left(\frac{\lambda_{j}^{p}}{\lambda_{i}^{p}}\right) \lambda_{j}^{p} \lambda_{i}^{p}}{\lambda_{j}^{p^{2}}-\lambda_{i}^{p^{2}}} & ; \text { otherwise }\end{cases}$

A Mises plastic potential on the Lagrangian axis of plastic stretch is used here for the associative $J_{2}$ flow of plasticity:

$\phi=\sqrt{\frac{3}{2} \overline{\bar{\eta}}_{L}: \overline{\bar{\eta}}_{L}}-\kappa=0$

in which $\kappa$ is a scalar parameter function of the equivalent plastic strain representing the current size of the yield surface.

With the assumption of maximum plastic dissipation (see for example Lemaitre and Chaboche [2] and Simo and Hughes [4]) the plastic strain rate tensor can be related to the normal to the yield surface as follows:

$\bar{d}^{p}=\dot{\alpha} \frac{\partial \phi}{\partial \bar{\tau}} \rightarrow \bar{d}_{E}^{p}=\dot{\alpha} \frac{\partial \phi}{\partial \overline{\bar{\tau}}_{L}}$ 
in which $\dot{\alpha}$ is the plastic multiplier which can be found from the consistency condition $\dot{\phi}=0$. The Kuhn-Tucker loading/unloading conditions [4] for the proposed model will therefore be as follows:

${\dot{\overline{E^{p}}}}_{L}=\dot{\alpha} h^{\log } \frac{\partial \phi}{\partial \overline{\bar{\tau}}_{L}}$

$\dot{\alpha} \geq 0 ; \phi \leq 0 ; \dot{\alpha} \phi=0$

The plastic spin $\bar{w}^{p}$ can be related to the known kinematics parameters and will be discussed in detail in the next section.

\section{4- Application of the proposed model to simple shear problem}

As shown in figure (3) a cube with the unit length is subjected to shear loading at the top edge while the bottom edge is fixed.

\section{Figure 3- Problem of simple shear}

The deformation gradient of this motion is given by:

$F=n_{1} \otimes n_{1}+n_{2} \otimes n_{2+} \gamma n_{1} \otimes n_{2}$

in which $\gamma$ is the applied shear. The polar decomposition of the deformation gradient yields the followings for the rigid rotation and the total left and right stretch tensors:

$$
\begin{aligned}
& V=\frac{1}{\sqrt{4+\gamma^{2}}}\left[\left(2+\gamma^{2}\right) n_{1} \otimes n_{1}+2 n_{2} \otimes n_{2+} \gamma\left(n_{1} \otimes n_{2}+n_{2} \otimes n_{1}\right)\right] \\
& U=\frac{1}{\sqrt{4+\gamma^{2}}}\left[2 n_{1} \otimes n_{1}+\left(2+\gamma^{2}\right) n_{2} \otimes n_{2+} \gamma\left(n_{1} \otimes n_{2}+n_{2} \otimes n_{1}\right)\right] \\
& R=\frac{1}{\sqrt{4+\gamma^{2}}}\left[2 n_{1} \otimes n_{1}+2 n_{2} \otimes n_{2+} \gamma\left(n_{1} \otimes n_{2}-n_{2} \otimes n_{1}\right)\right]
\end{aligned}
$$

The rigid spin of the material $\Omega^{R}$ is then given by:

$\Omega^{R}=\dot{R} R^{T}=\frac{2 \dot{\gamma}}{4+\gamma^{2}}\left(n_{1} \otimes n_{2}-n_{2} \otimes n_{1}\right)$

The rotated Lagrangian Kirchhoff stress tensor is given by:

$\overline{\bar{\tau}}_{L}=\overline{\bar{\tau}}_{L, 11}\left(n_{1} \otimes n_{1}-n_{2} \otimes n_{2}\right)+\overline{\bar{\tau}}_{L, 12}\left(n_{1} \otimes n_{2}+n_{2} \otimes n_{1}\right)$

Use of the proposed constitutive model given in equation (26) yields the following for the case of simple shear problem:

${\overline{\overline{U^{e}}}}_{L}=\exp \left(\frac{\overline{\bar{\tau}}_{L}}{2 G}\right)=\mathfrak{B} n_{1} \otimes n_{1}+\mathfrak{C} n_{2} \otimes n_{2+} \mathfrak{I}\left(n_{1} \otimes n_{2}+n_{2} \otimes n_{1}\right)$

in which $G$ is the shear modulus of the material and $\mathfrak{B}, \mathfrak{C}$, and $\mathfrak{J}$ are given by [34]: 


$$
\begin{aligned}
& \mathfrak{B}=\frac{1}{2 \mu \mathrm{T}}\left[\mathrm{T}\left(1+\mu^{2}\right)-\overline{\bar{\tau}}_{L, 11}\left(1-\mu^{2}\right)\right] \\
& \mathfrak{I}=\frac{1}{2 \mu \mathrm{T}}\left[\overline{\bar{\tau}}_{L, 12}\left(\mu^{2}-1\right)\right] \\
& \mathfrak{C}=\frac{1}{2 \mu \mathrm{T}}\left[\mathrm{T}\left(1+\mu^{2}\right)+\overline{\bar{\tau}}_{L, 11}\left(1-\mu^{2}\right)\right] \\
& \text { in which } \mathrm{T}=\sqrt{\overline{\bar{\tau}}_{L, 11}^{2}+\overline{\bar{\tau}}_{L, 12}^{2}} \text { and } \mu=\exp \left(\frac{\mathrm{T}}{2 G}\right) .
\end{aligned}
$$

The Mises plastic potential for kinematic hardening behaviour under simple shear is given by:

$$
\phi=\sqrt{3\left(\overline{\bar{\eta}}_{L, 11}^{2}+\overline{\bar{\eta}}_{L, 12}^{2}\right)}-\sigma_{Y}=0
$$

in which $\sigma_{Y}$ is the initial yield surface size and is assumed to be constant during plastic deformation.

Plastic incompressibility requires that the third invariant of the plastic stretch tensor be 1, i.e. $\operatorname{det} \chi^{p}=\operatorname{det} U^{p}=1$. Such an incompressibility condition specifies the following form for the diagonalized plastic stretch tensor for the case of the simple shear problem:

$\Lambda_{d}^{p}=\lambda^{p} n_{1} \otimes n_{1}+\frac{1}{\lambda^{p}} n_{2} \otimes n_{2}$

The rotation of the Lagrangian and Eulerian axes of the right plastic stretch tensor for the case of simple shear is given by:

$R_{L}^{p}=\cos \theta_{L}^{p}\left(n_{1} \otimes n_{1}+n_{2} \otimes n_{2}\right)+\sin \theta_{L}^{p}\left(n_{1} \otimes n_{2}-n_{2} \otimes n_{1}\right)$

$R_{E}^{p}=\cos \theta_{E}^{p}\left(n_{1} \otimes n_{1}+n_{2} \otimes n_{2}\right)+\sin \theta_{E}^{p}\left(n_{1} \otimes n_{2}-n_{2} \otimes n_{1}\right)$

in which $\theta_{L}^{p}$ and $\theta_{E}^{p}$ are the angles of the Lagrangian and Eulerian axes with respect to the fixed coordinate system, respectively.

Using the proposed decomposition given by equations (14) and (15), equations (20) and (25-2) yield the followings for the rotated Lagrangian elastic stretch:

${\overline{\overline{U^{e}}}}_{L}=R_{E}^{p^{T}} U R_{L}^{p} \Lambda_{d}^{p^{-1}}$

Taking the time derivative from both sides of equation (43) yields the followings for the components of time rate of the rotated Lagrangian elastic right stretch tensor:

$$
\begin{aligned}
& \frac{\mathrm{d} \overline{\bar{U}}_{L, 11}}{\mathrm{~d} \gamma}=\mathcal{F}_{1}\left(\lambda^{p}, \theta_{E}^{p}, \theta_{L}^{p} ; \gamma\right) \frac{\mathrm{d} \alpha}{\mathrm{d} \gamma}+\mathcal{G}_{1}\left(\lambda^{p}, \theta_{E}^{p}, \theta_{L}^{p} ; \gamma\right) \\
& \frac{\mathrm{d} \overline{\bar{U}_{L, 12}}}{\mathrm{~d} \gamma}=\mathcal{F}_{2}\left(\lambda^{p}, \theta_{E}^{p}, \theta_{L}^{p} ; \gamma\right) \frac{\mathrm{d} \alpha}{\mathrm{d} \gamma}+\mathcal{G}_{2}\left(\lambda^{p}, \theta_{E}^{p}, \theta_{L}^{p} ; \gamma\right)
\end{aligned}
$$

On the other hand, equations (38) and (39) yield the followings for the components of the time rate of the rotated Lagrangian elastic stretch tensor: 
$\frac{\dot{\overline{U^{e}}}}{L}=\frac{\mathrm{d}}{\mathrm{d} t}\left[\exp \left(\frac{\overline{\bar{\tau}}_{L}}{2 G}\right)\right]=\left[\dot{\mathfrak{B}} n_{1} \otimes n_{1}+\dot{\mathfrak{C}} n_{2} \otimes n_{2+} \dot{\mathfrak{I}}\left(n_{1} \otimes n_{2}+n_{2} \otimes n_{1}\right)\right]$

Therefore, the followings are derived for the material time rate of the Lagrangian rotated Kirchhoff stress with the help of equations (44) and (45):

$\frac{\mathrm{d} \overline{\bar{\tau}}_{L, 11}}{\mathrm{~d} \gamma}=\mathcal{A}_{1}\left(\lambda^{p}, \theta_{E}^{p}, \theta_{L}^{p}, \mathfrak{B}, \mathfrak{J}, \mathfrak{C} ; \gamma\right) \frac{\mathrm{d} \alpha}{\mathrm{d} \gamma}+\mathcal{B}_{1}\left(\lambda^{p}, \theta_{E}^{p}, \theta_{L}^{p}, \mathfrak{B}, \mathfrak{J}, \mathfrak{C} ; \gamma\right)$
$\frac{\mathrm{d} \overline{\bar{\tau}}_{L, 12}}{\mathrm{~d} \gamma}=\mathcal{A}_{2}\left(\lambda^{p}, \theta_{E}^{p}, \theta_{L}^{p}, \mathfrak{B}, \mathfrak{J}, \mathfrak{C} ; \gamma\right) \frac{\mathrm{d} \alpha}{\mathrm{d} \gamma}+\mathcal{B}_{2}\left(\lambda^{p}, \theta_{E}^{p}, \theta_{L}^{p}, \mathfrak{B}, \mathfrak{J}, \mathfrak{C} ; \gamma\right)$

The reader is referred to appendix 1 for a detail derivation of the component form of equations (44), (45), and (46) and their corresponding coefficients $\mathcal{F}_{1}, \mathcal{F}_{2}, \mathcal{G}_{1}, \mathcal{G}_{2}, \mathcal{A}_{1}, \mathcal{A}_{2}, \mathcal{B}_{1}$, and $\mathcal{B}_{2}$.

Using the proposed constitutive model for the evolution of the back stress tensor given by (28), the following differential equations are obtained for back stress tensor components:

$$
\begin{aligned}
\frac{\mathrm{d} \overline{\bar{\beta}}_{L, 11}}{\mathrm{~d} \gamma} & =\sqrt{\frac{3}{2}} H \overline{\bar{N}}_{L, 11} \frac{\mathrm{d} \alpha}{\mathrm{d} \gamma} \\
\frac{\mathrm{d} \overline{\bar{\beta}}_{L, 12}}{\mathrm{~d} \gamma} & =\sqrt{\frac{3}{2}} H h_{12}^{\log } \overline{\bar{N}}_{L, 12} \frac{\mathrm{d} \alpha}{\mathrm{d} \gamma}
\end{aligned}
$$

in which $\overline{\bar{N}}_{L}=\sqrt{\frac{2}{3}} \frac{\partial \phi}{\partial \overline{\bar{\eta}}_{L}}=\frac{\overline{\bar{\eta}}_{L}}{\left\|\overline{\bar{\eta}}_{L}\right\|}$ is the unit normal to the yield surface.

Use of equations (46) and (47) and the consistency condition which requires that $\dot{\phi}=0$ during plastic loading, the plastic multiplier can be obtained as follows:

$$
\frac{\mathrm{d} \alpha}{\mathrm{d} \gamma}=\frac{\overline{\bar{\eta}}_{L, 11} \mathcal{B}_{1}+\overline{\bar{\eta}}_{L, 12} \mathcal{B}_{2}}{\sqrt{\frac{3}{2}} H\left(\overline{\bar{N}}_{L, 11} \overline{\bar{\eta}}_{L, 11}+h_{12}^{\log } \overline{\bar{N}}_{L, 12} \overline{\bar{\eta}}_{L, 12}\right)-\left(\mathcal{A}_{1} \overline{\bar{\eta}}_{L, 11}+\mathcal{A}_{2} \overline{\bar{\eta}}_{L, 12}\right)}
$$

In summary, the governing differential equations for the problem of simple shear using the proposed constitutive model and the case of linear kinematic hardening are given as follows: 


$$
\begin{aligned}
& \frac{\mathrm{d} \overline{\bar{\tau}}_{L, 11}}{\mathrm{~d} \gamma}=\mathcal{A}_{1}\left(\lambda^{p}, \theta_{E}^{p}, \theta_{L}^{p}, \mathfrak{B}, \mathfrak{J}, \mathfrak{C} ; \gamma\right) \frac{\mathrm{d} \alpha}{\mathrm{d} \gamma}+\mathcal{B}_{1}\left(\lambda^{p}, \theta_{E}^{p}, \theta_{L}^{p}, \mathfrak{B}, \mathfrak{J}, \mathfrak{C} ; \gamma\right) \\
& \frac{\mathrm{d} \overline{\bar{\tau}}_{L, 12}}{\mathrm{~d} \gamma}=\mathcal{A}_{2}\left(\lambda^{p}, \theta_{E}^{p}, \theta_{L}^{p}, \mathfrak{B}, \mathfrak{I}, \mathfrak{C} ; \gamma\right) \frac{\mathrm{d} \alpha}{\mathrm{d} \gamma}+\mathcal{B}_{2}\left(\lambda^{p}, \theta_{E}^{p}, \theta_{L}^{p}, \mathfrak{B}, \mathfrak{I}, \mathfrak{C} ; \gamma\right) \\
& \frac{\mathrm{d} \overline{\bar{\beta}}_{L, 11}}{\mathrm{~d} \gamma}=\sqrt{\frac{3}{2}} H \overline{\bar{N}}_{L, 11} \frac{\mathrm{d} \alpha}{\mathrm{d} \gamma} \\
& \frac{\mathrm{d} \overline{\bar{\beta}}_{L, 12}}{\mathrm{~d} \gamma}=\sqrt{\frac{3}{2}} H h_{12}^{\log } \overline{\bar{N}}_{L, 12} \frac{\mathrm{d} \alpha}{\mathrm{d} \gamma} \\
& \frac{\mathrm{d} \theta_{E}^{p}}{\mathrm{~d} \gamma}=\mathfrak{T}_{1}\left(\lambda^{p}, \theta_{E}^{p}, \theta_{L}^{p} ; \gamma\right) \frac{\mathrm{d} \alpha}{\mathrm{d} \gamma}+\mathfrak{I}_{2}\left(\lambda^{p}, \theta_{E}^{p}, \theta_{L}^{p} ; \gamma\right) \\
& \frac{\mathrm{d} \theta_{L}^{p}}{\mathrm{~d} \gamma}=-\sqrt{\frac{3}{2}} \overline{\bar{N}}_{L, 12} \frac{2 \lambda^{p^{2}}}{p^{4}-1} \frac{\mathrm{d} \alpha}{\mathrm{d} \gamma} \\
& \frac{\mathrm{d} \lambda^{p}}{\mathrm{~d} \gamma}=\sqrt{\frac{3}{2}} \overline{\bar{N}}_{L, 11} \lambda^{p} \frac{\mathrm{d} \alpha}{\mathrm{d} \gamma} \\
& \frac{\mathrm{d} \alpha}{\mathrm{d} \gamma}=\frac{\overline{\bar{\eta}}_{L, 11} \mathcal{B}_{1}+\overline{\bar{\eta}}_{L, 12} \mathcal{B}_{2}}{\sqrt{\frac{3}{2}} H\left(\overline{\bar{N}}_{L, 11} \overline{\bar{\eta}}_{L, 11}+h_{12}^{\log } \overline{\bar{N}}_{L, 12} \overline{\bar{\eta}}_{L, 12}\right)-\left(\mathcal{A}_{1} \overline{\bar{\eta}}_{L, 11}+\mathcal{A}_{2} \overline{\bar{\eta}}_{L, 12}\right)}
\end{aligned}
$$

The evolution equation (49-5) is used for the update of the Eulerian triad angle during the time integration instead of equation (24) which needs the definition of the plastic spin. This is due to the fact that the plastic spin is a function of the known kinematics variables and does not require a separate evolution equation to be specified (see appendix 1 for detailed derivation of the evolution equations).

The set of differential equations given in (49) is numerically integrated for a maximum applied shear of $\gamma=8$ using a fourth-order Runge-Kutta numerical integration scheme. The amount of shear at which the plastic yielding starts is $\gamma_{p}=2 \sinh \left(\frac{\sigma_{Y}}{\sqrt{12} G}\right)$ and the initial conditions at this amount of shear are given as follows:

$$
\begin{aligned}
& \tau_{12}\left(\gamma_{p}\right)=\frac{4 G \operatorname{asinh}\left(\frac{\gamma_{p}}{2}\right)}{\sqrt{4+\gamma_{p}^{2}}} \\
& \tau_{11}\left(\gamma_{p}\right)=\sqrt{\frac{\sigma_{y}^{2}}{3}-\tau_{12}^{2}} \\
& \overline{\bar{\beta}}_{L, 11}\left(\gamma_{p}\right)=\overline{\bar{\beta}}_{L, 12}\left(\gamma_{p}\right)=0 \\
& \lambda^{p}\left(\gamma_{p}\right)=1 \\
& \theta_{E}^{p}\left(\gamma_{p}\right)=\theta_{L}^{p}\left(\gamma_{p}\right)=\theta\left(\gamma_{p}\right)=\operatorname{atan}\left(\frac{\gamma_{p}}{2}\right)
\end{aligned}
$$

Figures (4) and (5) show the evolution of the Kirchhoff stress using the proposed constitutive model for the problem of simple shear. Values of $\tau_{Y}=\sqrt{\frac{2}{3}} \sigma_{Y}=200 \mathrm{MPa}, H=\sqrt{\frac{2}{3}} \tau_{Y}$, and 
$G=\frac{30 \tau_{Y}}{\sqrt{6}}$ was used for the size of the yield surface, hardening modulus, and shear modulus of the material, respectively. The stress response of the same problem using the self-consistent Eulerian rate model of Bruhns et al. [10] based on the logarithmic (D) rate is also plotted. The stress responses of the original and modified formulations by Gabriel and Bathe $[29,30]$ as well as the stress response of the decoupled volumetric/deviatoric model of Simo [23,27], are also plotted for comparison. For a detail description of the original and modified formulation of Gabriel and Bathe the reader is referred to the Gabriel and Bathe [29] and Montans and Bathe [30]. The details of the hyper-based decoupled model of Simo and its numerical implementation can also be found in [23,27]. Figures (6) and (7) also show the evolution of the back stress components using the proposed constitutive model and models presented in $[10,23,27,29,30]$. It should be noted that the back stress components of the model proposed by Simo [23] shown in figure (6) and (7) are the decoupled deviatoric components used in the spatial representation of the model.

The response of the model is identical to those of the self-consistent Eulerian rate model of Bruhns et al. [10]. However, unlike the self-consistent Eulerian rate model of Bruhns et al. which is based on the specific logarithmic rate of the Kirchhoff stress, the proposed model is integrated without making any reference to any specific rate of stress. No objective rate of stress is used in the proposed model and a total hyperelastic stress function relates the Kirchhoff stress to the Hencky strain.

Figure (8) shows the evolution of the principal plastic stretches for the proposed model only.

Figure 4- Normal component of the Kirchhoff stress using different models

Figure 5- Shear component of the Kirchhoff stress using different models

Figure 6- Normal component of the back stress using different models

Figure 7- Shear component of the back stress using different models

Figure 8- Evolution of the principal plastic stretches (Proposed Model only) 


\section{5- Application of the proposed model for the prediction of mixed nonlinear hardening behaviour of SUS 304 stainless steel}

In this section the proposed constitutive model is extended to a mixed nonlinear kinematic/isotropic hardening. The model is then used to predict the behaviour of the SUS 304 stainless steel under fixed-end finite torsional loading.

With the help of the Armstrong-Frederick nonlinear kinematic hardening model [45], the proposed backstress evolution equation given in (28) can be modified as follows:

$\dot{\overline{\bar{\beta}}}_{L}=A_{f}{\dot{\overline{\bar{E}^{p}}}}_{L}-B_{f} \overline{\bar{\beta}}_{L} \dot{E}_{e q}^{p}$

in which $A_{f}$ and $B_{f}$ are the A-F material parameters and $\dot{E}_{e q}^{p}$ is the equivalent plastic strain rate which will be defined later in this section.

The Mises flow potential given in equation (31) is extended as follows for a nonlinear mixed hardening:

$\phi=\sqrt{\frac{3}{2} \overline{\bar{\eta}}_{L}: \overline{\bar{\eta}}_{L}}-\kappa=0$

in which $\kappa$ is a scalar valued function of the equivalent plastic strain which represents the current size of the yield surface and is related to the equivalent plastic strain through an exponential form as follows [46]:

$\kappa=\sigma_{Y 0}+\left(\sigma_{Y s}-\sigma_{Y 0}\right)\left[1-\exp \left(-b E_{e q}^{p}\right)\right]$

in which $\sigma_{Y 0}$ is the initial yield surface size, $\sigma_{Y S}$ is the saturation value for the subsequent yield stress, $b$ is a material parameter which controls the rate of saturation, and $E_{e q}^{p}=\int_{0}^{t} \dot{E}_{e q}^{p} \mathrm{~d} t$ is the accumulated equivalent plastic strain.

To derive a relation for the equivalent plastic strain, a modified plastic work is used here as follows:

$\dot{W}^{p}=\overline{\bar{\eta}}_{L}:{\dot{\overline{E^{p}}}}_{L}=\Sigma \dot{E}_{e q}^{p}$

in which $\Sigma=\sqrt{\frac{3}{2} \overline{\bar{\eta}}_{L}: \overline{\bar{\eta}}_{L}}=\kappa$. Equations (29) and (54) yield the following expression for the rate of the equivalent plastic strain for the case of the simple shear problem:

$\frac{\mathrm{d} E_{e q}^{p}}{\mathrm{~d} \gamma}=\frac{2\left(\overline{\bar{\eta}}_{L, 11}^{2}+h_{12}^{\log } \overline{\bar{\eta}}_{L, 12}^{2}\right)}{\left\|\overline{\bar{\eta}}_{L}\right\|^{2}} \frac{\mathrm{d} \alpha}{\mathrm{d} \gamma}$

Similar to the case of the linear kinematic hardening discussed in section 4 , the governing equations given in (49) are modified as follows for the case of the nonlinear mixed hardening. 
The evolution equations for the stress components remain the same as given in equations (49). The evolution equations for the back stress tensor should be modified as follows:

$$
\begin{aligned}
\frac{\mathrm{d} \overline{\bar{\beta}}_{L, 11}}{\mathrm{~d} \gamma} & =\left[\sqrt{\frac{3}{2}} A_{f} \overline{\bar{N}}_{L, 11}-\frac{2\left(\overline{\bar{\eta}}_{L, 11}^{2}+h_{12}^{\log } \overline{\bar{\eta}}_{L, 12}^{2}\right)}{\left\|\overline{\bar{\eta}}_{L}\right\|^{2}} B_{f} \overline{\bar{\beta}}_{L, 11}\right] \frac{\mathrm{d} \alpha}{\mathrm{d} \gamma} \\
\frac{\mathrm{d} \overline{\bar{\beta}}_{L, 12}}{\mathrm{~d} \gamma} & =\left[\sqrt{\frac{3}{2}} A_{f} h_{12}^{\log } \overline{\bar{N}}_{L, 12}-\frac{2\left(\overline{\bar{\eta}}_{L, 11}^{2}+h_{12}^{\log } \overline{\bar{\eta}}_{L, 12}^{2}\right)}{\left\|\overline{\bar{\eta}}_{L}\right\|^{2}} B_{f} \overline{\bar{\beta}}_{L, 12}\right] \frac{\mathrm{d} \alpha}{\mathrm{d} \gamma}
\end{aligned}
$$

And the consistency condition for the evolution of the plastic multiplier should be modified as follows:

$\frac{\mathrm{d} \alpha}{\mathrm{d} \gamma}=\frac{\overline{\bar{N}}_{L, 11} \mathcal{B}_{1}+\overline{\bar{N}}_{L, 12} \mathcal{B}_{2}}{T_{1}-T_{2}-T_{3}+T_{4}}$

in which:

$T_{1}=\sqrt{\frac{3}{2}} A_{f}\left(\overline{\bar{N}}_{L, 11} \overline{\bar{\eta}}_{L, 11}+h_{12}^{\log } \overline{\bar{N}}_{L, 12} \overline{\bar{\eta}}_{L, 12}\right)$

$T_{2}=\left(\mathcal{A}_{1} \overline{\bar{N}}_{L, 11}+\mathcal{A}_{2} \overline{\bar{N}}_{L, 12}\right)$

$T_{3}=\frac{2 B_{f}\left(\overline{\bar{\eta}}_{L, 11}^{2}+h_{12}^{\log } \overline{\bar{\eta}}_{L, 12}^{2}\right)}{\left\|\overline{\bar{\eta}}_{L}\right\|^{2}}\left(\overline{\bar{\beta}}_{L, 11} \overline{\bar{\eta}}_{L, 11}+\overline{\bar{\beta}}_{L, 12} \overline{\bar{\eta}}_{L, 12}\right)$

$T_{4}=\frac{b\left(\sigma_{Y s}-\kappa\right)}{\sqrt{6}}$

Similar to the case of linear kinematic hardening model, the governing equations given by (49) with their corresponding modified equations given by equations (52) to (58) are numerically integrated using the fourth-order Runge-Kutta method for a maximum applied shear of $\gamma=4$. The stress responses from the proposed model are plotted in figure (9) using the material parameters given in [35] for the SUS 304 stainless steel which are summarized in table (1) below. The model prediction for the fixed-end finite torsional loading of the SUS 304 is in good agreement with the experimental data reported by Ishikawa [35]. Furthermore, from figure (9), the proposed model gives identical results to those of the self-consistent Eulerian model of Bruhns et al. [10], based on the logarithmic (D) rate. Figures (10) and (11) also show the evolution of the back stress tensor and subsequent yield surface size vs. the applied shear, respectively. 


\begin{tabular}{|l|l|}
\hline Shear Modulus & $G=78(\mathrm{GPa})$ \\
\hline Exponential Isotropic Hardening Parameters & $\sigma_{Y 0}=285.6(\mathrm{MPa}) ; \sigma_{Y S}=680(\mathrm{MPa}) ; b=\frac{5}{3}$ \\
\hline Armstrong-Frederick Model Parameters & $A_{f}=20(\mathrm{MPa}) ; B_{f}=0.2$ \\
\hline
\end{tabular}

Table 1- Parameters used for the mixed hardening behaviour of SUS 304 [35]

Figure 9- Stress components for SUS 304 stainless steel under fixed-end finite torsional loading using the proposed mixed hardening model, self-consistent model based on logarithmic rate, and experimental data

Figure 10- Evolution of back stress components for SUS 304 stainless steel under fixed-end torsion using the proposed mixed hardening model and self-consistent model based on logarithmic rate

Figure 11- Evolution of subsequent yield surface size for SUS 304 stainless steel under fixed-end torsion using the proposed mixed hardening model and the self-consistent model based on the logarithmic rate

\section{6- Conclusions}

A new constitutive model based on a hyperelastic function was proposed for the finite elastoplastic deformation of the hardening materials. A modified class of multiplicative decomposition of the deformation gradient was proposed and used in the model. The plastic right stretch tensor and its corresponding Lagrangian axis were used for model integration. Furthermore, a new model for the evolution of the back stress tensor was proposed on the Lagrangian axis of the plastic right stretch tensor.

The problem of simple shear was solved for the case of linear kinematic hardening. Results obtained were identical to those of the self-consistent Eulerian rate model of Bruhns et al. [10] based on the logarithmic (D) rate. Unlike the hypo-based Eulerian rate model of elastoplasticity, the proposed model does not employ any objective rate of stress.

The proposed constitutive equation was extended to a mixed nonlinear kinematic/isotropic hardening. The governing equations were integrated using the material parameters for SUS 304 stainless steel. Results were in good agreement with those of the experimental results reported by Ishikawa [35] for the fixed-end finite torsional loading of this material.

The proposed model is simple and can be used for finite deformation analysis of elastoplastic materials. Since the model refers to no rate of stress for its integration, the need for a neutrally objective time integration algorithm is bypassed. As a result, the model can be efficiently implemented for the strain space formulation of finite element method. 


\section{Acknowledgment}

The financial support of the Natural Sciences and Engineering Research Council of Canada, NSERC, is gratefully acknowledged.

\section{Appendix 1}

To derive a relation between the time rate of stress and plastic multiplier to be used for plastic integration and satisfying plastic consistency condition for the problem of simple shear, equations (38) and (39) yield:

${\dot{\overline{U^{e}}}}_{L}=\frac{\mathrm{d}}{\mathrm{d} t}\left[\exp \left(\frac{\overline{\bar{\tau}}_{L}}{2 G}\right)\right]=\left[\dot{\mathfrak{B}} n_{1} \otimes n_{1}+\dot{\mathfrak{C}} n_{2} \otimes n_{2+} \dot{\mathfrak{I}}\left(n_{1} \otimes n_{2}+n_{2} \otimes n_{1}\right)\right]$

in which:

$\dot{\mathfrak{B}}=\left(v_{1} \overline{\bar{\tau}}_{L, 11}-v_{0}\right) \dot{\overline{\bar{\tau}}}_{L, 11}+v_{1} \overline{\bar{\tau}}_{L, 12} \dot{\overline{\bar{\tau}}}_{L, 12}$
$\dot{\mathfrak{J}}=v_{2} \overline{\bar{\tau}}_{L, 11} \dot{\overline{\bar{\tau}}}_{L, 11}+\left(v_{2} \overline{\bar{\tau}}_{L, 12}-v_{0}\right) \dot{\overline{\bar{\tau}}}_{L, 12}$
$\dot{\mathfrak{T}}=\left(v_{3} \overline{\bar{\tau}}_{L, 11}+v_{0}\right) \dot{\overline{\bar{\tau}}}_{L, 11}+v_{3} \overline{\bar{\tau}}_{L, 12} \dot{\overline{\bar{\tau}}}_{L, 12}$

and the scalar $v_{i}$ 's are given as follows:

$v_{0}=\frac{1-\mu^{2}}{2 \mu \mathrm{T}}$

$v_{1}=\frac{1}{2 \mu G \mathrm{~T}^{2}}\left[G\left(1+\mu^{2}\right)+\mu^{2}\left(\mathrm{~T}+\overline{\bar{\tau}}_{L, 11}\right)-\mathfrak{B} \mu(2 \mathrm{G}+\mathrm{T})\right]$

$v_{2}=\frac{1}{2 G \mathrm{~T}^{2}}\left[\mu \overline{\bar{\tau}}_{L, 12}-\mathfrak{\Im}(2 \mathrm{G}+\mathrm{T})\right]$

$v_{3}=\frac{1}{2 \mu G \mathrm{~T}^{2}}\left[G\left(1+\mu^{2}\right)+\mu^{2}\left(\mathrm{~T}-\overline{\bar{\tau}}_{L, 11}\right)-\mathfrak{C} \mu(2 \mathrm{G}+\mathrm{T})\right]$

Use of equations (38), (39), and (43) yields:

${\overline{\overline{U^{e}}}}_{L}=R_{E}^{p^{T}} U R_{L}^{p} \Lambda_{d}^{p^{-1}}=\exp \left(\frac{\overline{\bar{\tau}}_{L}}{2 G}\right)=\mathfrak{B} n_{1} \otimes n_{1}+\mathfrak{C} n_{2} \otimes n_{2+} \mathfrak{J}\left(n_{1} \otimes n_{2}+n_{2} \otimes n_{1}\right)$

Taking the time derivative of (A-4) results into the followings for time rate of $\overline{\overline{U^{e}}}{ }_{L}$ :

${\overline{\overline{U^{e}}}}_{L}=\left(-\Omega_{E}^{p}+R_{E}^{p^{T}} \dot{U} U^{-1} R_{E}^{p}+R_{E}^{p^{T}} U \Omega_{L}^{p} U^{-1} R_{E}^{p}\right){\overline{\overline{U^{e}}}}_{L}+{\overline{\overline{U^{e}}}}_{L} \Lambda_{d}^{p} \dot{\Lambda}_{d}^{p^{-1}}$

Substituting for known kinematics quantities such as $U, \dot{U}$, and $U^{-1}$ in (A-5) and knowing that $\frac{\mathrm{d} \Omega_{E}^{p}}{\mathrm{~d} \gamma}=\frac{\mathrm{d} \theta_{E}^{p}}{\mathrm{~d} \gamma}\left(n_{1} \otimes n_{2}-n_{2} \otimes n_{1}\right)$, and $\frac{\mathrm{d} \Omega_{L}^{p}}{\mathrm{~d} \gamma}=-\frac{\sqrt{6} \lambda^{2} \overline{\bar{N}}_{L, 12}}{\lambda^{p^{4}}-1} \frac{\mathrm{d} \alpha}{\mathrm{d} \gamma}\left(n_{1} \otimes n_{2}-n_{2} \otimes n_{1}\right)$, the followings are obtained for the components of the time derivative of the rotated elastic stretch tensor on the Lagrangian axis: 


$$
\begin{aligned}
& \frac{\mathrm{d} \overline{\bar{U}}_{L, 11}}{\mathrm{~d} \gamma}=-\mathfrak{J} \frac{\mathrm{d} \theta_{E}^{p}}{\mathrm{~d} \gamma}-\left[\frac{\sqrt{6} \lambda^{p^{2}} \overline{\bar{N}}_{L, 12}}{\lambda^{p^{4}}-1}\left(M_{1} L_{1}+M_{2} L_{3}\right)+\sqrt{\frac{3}{2}} \mathfrak{B} \overline{\bar{N}}_{L, 11}\right] \frac{\mathrm{d} \alpha}{\mathrm{d} \gamma}+\left(K_{1} L_{1}+K_{2} L_{3}\right) \\
& \frac{\mathrm{d} \overline{\bar{U}}_{L, 12}}{\mathrm{~d} \gamma}=-\mathfrak{C} \frac{\mathrm{d} \theta_{E}^{p}}{\mathrm{~d} \gamma}+\left[-\frac{\sqrt{6} \lambda^{2} \overline{\bar{N}}_{L, 12}}{\lambda^{p^{4}}-1}\left(M_{1} L_{2}+M_{2} L_{4}\right)+\sqrt{\frac{3}{2}} \mathfrak{\Im} \overline{\bar{N}}_{L, 11}\right] \frac{\mathrm{d} \alpha}{\mathrm{d} \gamma}+\left(K_{1} L_{2}+K_{2} L_{4}\right) \\
& \frac{\mathrm{d} \overline{\bar{U}}_{L, 21}}{\mathrm{~d} \gamma}=\mathfrak{B} \frac{\mathrm{d} \theta_{E}^{p}}{\mathrm{~d} \gamma}-\left[\frac{\sqrt{6} \lambda^{2} \overline{\bar{N}}_{L, 12}}{\lambda^{p^{4}}-1}\left(M_{3} L_{1}+M_{4} L_{3}\right)+\sqrt{\frac{3}{2}} \mathfrak{\Im} \overline{\bar{N}}_{L, 11}\right] \frac{\mathrm{d} \alpha}{\mathrm{d} \gamma}+\left(K_{3} L_{1}+K_{4} L_{3}\right)
\end{aligned}
$$

in which:

$$
\begin{aligned}
& K_{1}\left(\theta_{E}^{p} ; \gamma\right)=-g_{1}(\gamma) \cos \theta_{E}^{p}-g_{3}(\gamma) \sin \theta_{E}^{p} \\
& K_{2}\left(\theta_{E}^{p} ; \gamma\right)=g_{2}(\gamma) \cos \theta_{E}^{p}-g_{1}(\gamma) \sin \theta_{E}^{p} \\
& K_{3}\left(\theta_{E}^{p} ; \gamma\right)=-g_{1}(\gamma) \sin \theta_{E}^{p}+g_{3}(\gamma) \cos \theta_{E}^{p} \\
& K_{4}\left(\theta_{E}^{p} ; \gamma\right)=g_{2}(\gamma) \sin \theta_{E}^{p}+g_{1}(\gamma) \cos \theta_{E}^{p} \\
& g_{1}(\gamma)=\frac{2 \gamma}{4+\gamma^{2}} ; g_{2}(\gamma)=\frac{2}{4+\gamma^{2}} ; g_{3}(\gamma)=\frac{8-\gamma^{2}\left(2+\gamma^{2}\right)}{\left(4+\gamma^{2}\right)^{2}} \\
& M_{1}\left(\theta_{E}^{p} ; \gamma\right)=-\gamma \cos \theta_{E}^{p}+\left(1+\gamma^{2}\right) \sin \theta_{E}^{p} \\
& M_{2}\left(\theta_{E}^{p} ; \gamma\right)=\cos \theta_{E}^{p}-\gamma \sin \theta_{E}^{p} \\
& M_{3}\left(\theta_{E}^{p} ; \gamma\right)=-\gamma \sin \theta_{E}^{p}-\left(1+\gamma^{2}\right) \cos \theta_{E}^{p} \\
& M_{4}\left(\theta_{E}^{p} ; \gamma\right)=\sin \theta_{E}^{p}+\gamma \cos \theta_{E}^{p}
\end{aligned}
$$

and:

$L_{1}\left(\mathfrak{B}, \mathfrak{I}, \mathfrak{C}, \theta_{E}^{p}\right)=\mathfrak{B} \cos \theta_{E}^{p}+\mathfrak{I} \sin \theta_{E}^{p}$

$L_{2}\left(\mathfrak{B}, \mathfrak{J}, \mathfrak{C}, \theta_{E}^{p}\right)=\mathfrak{J} \cos \theta_{E}^{p}+\mathfrak{C} \sin \theta_{E}^{p}$

$L_{3}\left(\mathfrak{B}, \mathfrak{J}, \mathfrak{C}, \theta_{E}^{p}\right)=-\mathfrak{B} \sin \theta_{E}^{p}+\mathfrak{I} \cos \theta_{E}^{p}$

$L_{4}\left(\mathfrak{B}, \mathfrak{J}, \mathfrak{C}, \theta_{E}^{p}\right)=-\mathfrak{I} \sin \theta_{E}^{p}+\mathfrak{C} \cos \theta_{E}^{p}$

Equations (A-7) and (A-8) can be used to find a relation for the evolution of the $\theta_{E}^{p}$ during plastic loading. Symmetry of $\overline{\bar{U}^{e}}{ }_{L}$ requires that ${\overline{\overline{U^{e}}}}_{L}$ be also symmetric; as a result, $\frac{\mathrm{d}{\overline{\overline{U^{e}}}}_{L, 21}}{\mathrm{~d} \gamma}=$ $\frac{\mathrm{d} \overline{\bar{U}}_{L, 12}}{\mathrm{~d} \gamma}$ which leads to:

$$
\begin{aligned}
& \frac{\mathrm{d} \theta_{E}^{p}}{\mathrm{~d} \gamma}=\frac{1}{\mathfrak{B}+\mathfrak{C}}\left(K_{1} L_{2}+K_{2} L_{4}-K_{3} L_{1}-K_{4} L_{3}\right)+\frac{\sqrt{6}}{\mathfrak{B}+\mathfrak{C}}\left\{\widetilde{\mathfrak{S}}_{L, 11}+\frac{\overline{\bar{N}}_{L, 12} \lambda^{p^{2}}}{\lambda^{p^{4}}-1}\left(M_{3} L_{1}+M_{4} L_{3}-M_{1} L_{2}-\right.\right. \\
& \left.\left.M_{2} L_{4}\right)\right\} \frac{\mathrm{d} \alpha}{\mathrm{d} \gamma}
\end{aligned}
$$

Or equivalently:

$$
\frac{\mathrm{d} \theta_{E}^{p}}{\mathrm{~d} \gamma}=\mathfrak{I}_{1} \frac{\mathrm{d} \alpha}{\mathrm{d} \gamma}+\mathfrak{I}_{2}
$$


Substituting (A-12) into (A-6) and (A-7) yields:

$$
\begin{aligned}
& \frac{\mathrm{d}{\overline{\overline{U^{e}}}}_{L, 11}}{\mathrm{~d} \gamma}=-\left[\mathfrak{J}_{\mathfrak{I}_{1}}+\frac{\sqrt{6} \lambda^{p^{2}} \overline{\bar{N}}_{L, 12}}{\lambda^{p^{4}}-1}\left(M_{1} L_{1}+M_{2} L_{3}\right)+\sqrt{\frac{3}{2}} \mathfrak{B} \overline{\bar{N}}_{L, 11}\right] \frac{\mathrm{d} \alpha}{\mathrm{d} \gamma}+\left(K_{1} L_{1}+K_{2} L_{3}-\mathfrak{J} \mathfrak{I}_{2}\right) \\
& \frac{\mathrm{d} \overline{\bar{U}}_{L, 12}}{\mathrm{~d} \gamma}=\left[-\mathfrak{C I}_{1}-\frac{\sqrt{6} \lambda^{2} \overline{\bar{N}}_{L, 12}}{\lambda^{p^{4}}-1}\left(M_{1} L_{2}+M_{2} L_{4}\right)+\sqrt{\frac{3}{2}} \mathfrak{\Im} \overline{\bar{N}}_{L, 11}\right] \frac{\mathrm{d} \alpha}{\mathrm{d} \gamma}+\left(K_{1} L_{2}+K_{2} L_{4}-\mathfrak{C} \mathfrak{I}_{2}\right)
\end{aligned}
$$

Or equivalently:

$\frac{\mathrm{d}{\overline{\overline{U^{e}}}}_{L, 11}}{\mathrm{~d} \gamma}=\mathcal{F}_{1}\left(\lambda^{p}, \theta_{E}^{p}, \mathfrak{B}, \mathfrak{I}, \mathfrak{C}, \overline{\bar{N}}_{L, 11}, \overline{\bar{N}}_{L, 12} ; \gamma\right) \frac{\mathrm{d} \alpha}{\mathrm{d} \gamma}+\mathcal{G}_{1}\left(\lambda^{p}, \theta_{E}^{p}, \mathfrak{B}, \mathfrak{I}, \mathfrak{C}, \overline{\bar{N}}_{L, 11}, \overline{\bar{N}}_{L, 12} ; \gamma\right)$
$\frac{\mathrm{d}{\overline{\overline{U^{e}}}}_{L, 12}}{\mathrm{~d} \gamma}=\mathcal{F}_{2}\left(\lambda^{p}, \theta_{E}^{p}, \mathfrak{B}, \mathfrak{I}, \mathfrak{C}, \overline{\bar{N}}_{L, 11}, \overline{\bar{N}}_{L, 12} ; \gamma\right) \frac{\mathrm{d} \alpha}{\mathrm{d} \gamma}+\mathcal{G}_{2}\left(\lambda^{p}, \theta_{E}^{p}, \mathfrak{B}, \mathfrak{I}, \mathfrak{C}, \overline{\bar{N}}_{L, 11}, \overline{\bar{N}}_{L, 12} ; \gamma\right)$

It is worth mentioning that use of a definition for the plastic spin is bypassed due to the symmetry property of the elastic stretch tensor. In other words, in isotropic plasticity the plastic spin is function of the known kinematics variables and does not require a separate evolution equation (see for example $[33,41])$.

Using equations (A-2) and (A-15) gives the followings for the time rate of stress tensors:

$$
\begin{aligned}
& \dot{\mathfrak{B}}=\left(v_{1} \overline{\bar{\tau}}_{L, 11}-v_{0}\right) \dot{\bar{\tau}}_{L, 11}+v_{1} \overline{\bar{\tau}}_{L, 12} \dot{\overline{\bar{\tau}}}_{L, 12}=\mathcal{F}_{1} \frac{\mathrm{d} \alpha}{\mathrm{d} \gamma}+\mathcal{G}_{1} \\
& \dot{\mathfrak{I}}=v_{2} \overline{\bar{\tau}}_{L, 11} \dot{\bar{\tau}}_{L, 11}+\left(v_{2} \overline{\bar{\tau}}_{L, 12}-v_{0}\right) \dot{\overline{\bar{\tau}}}_{L, 12}=\mathcal{F}_{2} \frac{\mathrm{d} \alpha}{\mathrm{d} \gamma}+\mathcal{G}_{2}
\end{aligned}
$$

Therefore, equation (A-16) yields the following expression for the time rate of stress components:

$$
\begin{aligned}
& \frac{\mathrm{d} \overline{\bar{\tau}}_{L, 11}}{\mathrm{~d} \gamma}=\mathcal{A}_{1}\left(\lambda^{p}, \theta_{E}^{p}, \theta_{L}^{p}, \mathfrak{B}, \mathfrak{J}, \mathfrak{C} ; \gamma\right) \frac{\mathrm{d} \alpha}{\mathrm{d} \gamma}+\mathcal{B}_{1}\left(\lambda^{p}, \theta_{E}^{p}, \theta_{L}^{p}, \mathfrak{B}, \mathfrak{J}, \mathfrak{C} ; \gamma\right) \\
& \frac{\mathrm{d} \overline{\bar{\tau}}_{L, 12}}{\mathrm{~d} \gamma}=\mathcal{A}_{2}\left(\lambda^{p}, \theta_{E}^{p}, \theta_{L}^{p}, \mathfrak{B}, \mathfrak{J}, \mathfrak{C} ; \gamma\right) \frac{\mathrm{d} \alpha}{\mathrm{d} \gamma}+\mathcal{B}_{2}\left(\lambda^{p}, \theta_{E}^{p}, \theta_{L}^{p}, \mathfrak{B}, \mathfrak{J}, \mathfrak{C} ; \gamma\right)
\end{aligned}
$$

in which:

$\left[\begin{array}{ll}\mathcal{A}_{1} & \mathcal{B}_{1} \\ \mathcal{A}_{2} & \mathcal{B}_{2}\end{array}\right]=\left[\begin{array}{cc}v_{1} \overline{\bar{\tau}}_{L, 11}-v_{0} & v_{1} \overline{\bar{\tau}}_{L, 12} \\ v_{2} \overline{\bar{\tau}}_{L, 11} & v_{2} \overline{\bar{\tau}}_{L, 12}-v_{0}\end{array}\right]^{-1}\left[\begin{array}{cc}\mathcal{F}_{1} & \mathcal{G}_{1} \\ \mathcal{F}_{2} & \mathcal{G}_{2}\end{array}\right]$

Equations (A-12) and (A-18) are used during the time integration for the plastic consistency and update of the Eulerian triad angle for the problem of simple shear. 


\section{References}

[1] Khan A.S., Huang S. Continuum theory of plasticity. New York: Wiley; 1995.

[2] Lemaitre J., Chaboche J.L. Mechanics of Solid Materials, UK: Cambridge University Press; 1994.

[3] Swift W., Length changes in metals under torsional overstrain. Engineering 1947; 163:253-257.

[4] Simo J.C., Hughes T.J.R. Computational inelasticity. Berlin: Springer; 1998.

[5] Green A.E., Naghdi P.M. A general theory of an elastic-plastic continuum. Arch Rat Mech Anal $1965 ; 18: 251-81$.

[6] Sansour C., Wagner W., Viscoplasticity based on additive decomposition of logarithmic strain and unified constitutive equations, Theoretical and computational considerations with reference to shell applications. Computers and Structures 2003; 81:1583-1594.

[7] Nemat-Nasser S. Decomposition of strain measures and their rates in finite deformation elastoplasticity. Int. J. Solids Struct 1979; 15:155-166.

[8] Nemat-Nasser S. On finite deformation elasto-plasticity. Int J Solids Struct 1982; 18:857-72.

[9] Dafalias Y.F. Corotational rates for kinematic hardening at large plastic deformations. ASME J. Appl. Mech. 1983; 561-565.

[10] Bruhns O.T., Xiao H., Meyers A. Self-consistent Eulerian rate type elasto-plasticity models based upon the logarithmic stress rate. 1999; 15:479-520.

[11] Truesdell C., Noll W. The non-linear field theories of mechanics, third edition. Berlin: Springer; 2003.

[12] Truesdell C. The simplest rate theory of pure elasticity. Comm. Pure Appl. Math. 1955; 8:123132.

[13] Nagtegaal J.C., de Jong J.E. Some aspects of non-isotropic work-hardening in finite strain plasticity. In: Proceeding of the Workshop on Plasticity of Metals at Finite Strain: Theory, Experimental and Computation, Stanford University 1981; 65-102.

[14] Koji M., Bathe K.J. Studies of finite element procedures-stress solution of a closed elastic strain path with stretching and shearing using the updated Lagrangian Jaumann formulation. Comput. Struct. 1987; 26:175-179.

[15] Bernstein B. Relations between hypo-elasticity and elasticity. Transactions of the Society of Rheology. 1960; IV: 23-28.

[16] Ericksen J.L. Hypo-elastic potentials. Quart. Journ. Mech. And Applied Math. 1958; Vol. XI, Pt. 1.

[17] Lehmann T., Guo Z.H., Liang H.Y. The conjugacy between Cauchy stress and logarithm of the left stretch tensor. Eur. J. Mech. A/Solids. 1991; 10:395-404. 
[18] Reinhardt W.D., Dubey R.N. Application of Objective Rates in Mechanical Modeling of Solids. J. Applied Mechanics (Trans. ASME) 1996; 118:692-698.

[19] Xiao H., Bruhns O.T., Meyers A. Logarithmic strain, logarithmic spin and logarithmic rate. Acta Mechanica. 1997; 124:89-105.

[20] Lee E.H. Elastic-plastic deformations at finite strains. Journal of Applied Mechanics (ASME) 1969; 36:1-6.

[21] Lubarda V.A. Duality in constitutive formulation of finite-strain elastoplasticity based on $F=F_{e} F_{p}$ and $\mathrm{F}=\mathrm{F}_{\mathrm{p}} \mathrm{F}_{\mathrm{e}}$ decompositions. Int J Plasticity 1999; 15:1277-90.

[22] Asaro R.J. Micromechanics of crystals and polycrystals. Advances in Applied Mechanics 1983; 23:1-115.

[23] Simo J.C., A framework for finite strain elastoplasticity based on maximum plastic dissipation and the multiplicative decomposition: Part I. Continuum Formulation. Computer Methods in Applied Mechanics and Engineering 1988; 66:199-219.

[24] Simo J.C., Ortiz M. A unified approach to finite deformation elastoplastic analysis based on the use of hyperelastic constitutive equations. Computer Methods in Applied Mechanics and Engineering 1985; 49:221-245.

[25] Ogden R.W. Elastic deformations in rubberlike solids, in: H.G. Hopkins and M.J. Sewell, eds., Mechanics of solids, The Rodney Hill 60th Anniversary Volume 1982; 499-537.

[26] Ogden R.W. Nonlinear elastic deformations. Ellis Horwood West Sussex, U.K. 1984.

[27] Simo J.C., A framework for finite strain elastoplasticity based on maximum plastic dissipation and the multiplicative decomposition: Part II. Computational aspects. Computer Methods in Applied Mechanics and Engineering 1988; 68:1-31.

[28] Weber G., Anand L. Finite Deformation Constitutive Equations and a Time Integration Procedure for Isotropic Hyperelastic-Viscoplastic Solids. Computer Methods in Applied Mechanics and Engineering 1990; 79:173-202.

[29] Gabriel G., Bathe K.J. Some Computational Issues in Large Strain Elasto-Plastic Analysis. Computers \& Structures 1995; 56:249-267.

[30] Montans F.J., Bathe K.J. Computational issues in large strain elasto-plasticity: an algorithm for mixed hardening and plastic spin. International Journal for Numerical Methods in Engineering 2005; 63:159-196

[31] Anand L. Moderate deformations in extension-torsion of incompressible isotropic elastic materials. Journal of the Mechanics and Physics of Solids 1986; 34:293-304.

[32] Anand L. On H. Hencky's approximate strain-energy function for moderate deformations. Journal of Applied Mechanics (ASME) 1979; 46:78-82. 
[33] Metzger D.R., Dubey R.N. Corotational rates in constitutive modeling of elastic-plastic deformation. Int. J. Plasticity 1987; 4:341-368.

[34] Ghavam K., Naghdabadi R. Hardening materials modeling in finite elastic-plastic deformations based on the stretch tensor decomposition. Materials and Design 2008; 29:161-172.

[35] Ishikawa H. Constitutive model of plasticity in finite deformation. International Journal of Plasticity 1999; 15:299-317.

[36] Rivlin R.S. Further remarks on the stress deformation relations for isotropic materials. J. Rat. Mech. Anal. 1955; 4:702.

[37] Diens J.K. On the analysis of rotation and stress rate in deforming bodies. Acta Mech 1979; 32:217-232.

[38] Xiao H., Bruhns O.T., Meyers A. The integrability criterion in finite elastoplasticity and its constitutive implications. Acta Mechanica 2007; 188:227-244.

[39] Marsden J.E., Hughes T.J.R. Mathematical foundations of elasticity. New York: Dover; 1994.

[40] Mandel J. Thermodynamics and plasticity. In Foundations of Continuum Thermodynamics, Delgado JJ, Nina NR, Whitelaw JH (eds). Macmillan: London, 1974; 283-304.

[41] Reinhardt W.D., Dubey R.N. An Eulerian-based approach to elastic-plastic decomposition. Acta Mechanica 1998; 131:111-119.

[42] Hill R. Constitutive inequalities for isotropic elastic solids under finite strain. Proc. R. Soc. A 1970; 314:457-472.

[43] Eterovic A.L., Bathe K.J. A note on the use of the additive decomposition of the strain tensor in finite deformation inelasticity. Computer Methods in Applied Mechanics and Engineering 1991; 93:31-38.

[44] Hoger A. The stress conjugate to logarithmic strain. Int. J. Solids Structures 1987; 23:1645-1656

[45] Armstrong P.J., Frederick O.C. A mathematical representation of the multiaxial Bauschinger effect. CEGB report No. RD/B/N731 1996.

[46] Voce E. "Metallurgica", 1955; Col. 51, pp. 219. 